\title{
Efficacy, safety and patient-reported outcomes of combination etanercept and sulfasalazine versus etanercept alone in patients with rheumatoid arthritis: a double-blind randomised 2-year study
}

\author{
B Combe, ${ }^{1}$ C Codreanu, ${ }^{2}$ U Fiocco, ${ }^{3}$ M Gaubitz, ${ }^{4}$ P P Geusens, ${ }^{5,6}$ T K Kvien, ${ }^{7}$ K Pavelka, ${ }^{8}$ \\ P N Sambrook, ${ }^{9}$ J S Smolen, ${ }^{10}$ R Khandker, ${ }^{11}$ A Singh, ${ }^{11} \mathrm{~J}$ Wajdula, ${ }^{11}$ S Fatenejad, ${ }^{11}$ for \\ the Etanercept European Investigators Network ${ }^{*}$
}

${ }^{1}$ Service d'ImmunoRhumatologie, Hopital Lapeyronie, Montpellier, France; ${ }^{2}$ Centrul Metodologic de Reumatologie, Bucuresti, Romania; ${ }^{3}$ Cattedra e Divisione di Reumatologia, Policlinico Universitario, Padova, Italy:

${ }^{4}$ Medical Clinic B Westfalian-

Wilhelms-Univ, Munster,

Germany; ${ }^{5}$ Biomedical Research

Center, University Hasselt,

Hasselt, Belgium; ${ }^{6}$ Department

of Internal Medicine/

Rheumatology, University

Maastricht, Maastricht, The

Netherlands; ${ }^{7}$ Department of

Rheumatology, Diakonhjemmets

Hospital, Oslo, Norway;

${ }^{8}$ Institute of Rheumatology,

Praha, Czech Republic; ${ }^{9}$ Kolling Institute, University of Sydney,

Sydney, Australia; ${ }^{10}$ 2nd

Department of Medicine,

Krankenhaus Lainz and

Department of Rheumatology,

Internal Medicine III, Medical

University of Vienna, Vienna,

Austria; ${ }^{11}$ Wyeth Research,

Collegeville, Pennsylvania, USA

Correspondence to:

Dr J Wajdula, Clinical Research and Development, Wyeth

Research, 500 Arcola Road,

Collegeville, PA 19426, USA

wajdulj@wyeth.com

*Etanercept Study 309 investigators are listed at the end of the paper

Accepted 17 August 2008

Published Online First

11 September 2008

\begin{abstract}
Objective: To determine the efficacy and safety of etanercept and etanercept plus sulfasalazine versus sulfasalazine in patients with rheumatoid arthritis (RA) despite sulfasalazine therapy.
\end{abstract}

Methods: Patients were randomly assigned to etanercept (25 mg twice weekly; sulfasalazine was discontinued at baseline), etanercept plus sulfasalazine (unchanged regimen of 2-3 g/day) or sulfasalazine in a double-blind, randomised, 2-year study in adult patients with active RA despite sulfasalazine therapy. Efficacy was assessed using the American College of Rheumatology criteria, disease activity scores (DAS) and patient-reported outcomes (PRO).

Results: Demographic variables and baseline disease characteristics were comparable among treatment groups; mean DAS 5.1, 5.2 and 5.1 for etanercept ( $n=103$ ), etanercept plus sulfasalazine $(n=101)$ and sulfasalazine ( $n=50$ ), respectively. Withdrawal due to lack of efficacy was highest with sulfasalazine (26 (52\%) vs $6(6 \%)$ for either etanercept group, $p<0.001)$. Patients receiving etanercept or etanercept plus sulfasalazine had a more rapid initial response, which was sustained at 2 years, than those receiving sulfasalazine: mean DAS 2.8, 2.5 versus 4.5, respectively $(p<0.05)$; ACR 20 response was achieved by $67 \%, 77 \%$ versus $34 \%$ of patients, respectively $(p<0.01)$ Overall, PRO followed a similar pattern; a clinically significant improvement in health assessment questionnaire was achieved by $76 \%, 78 \%$ versus $40 \%$ of patients, respectively $(p<0.01)$. Commonly reported adverse events occurring in the etanercept groups were injection site reactions and pharyngitis/laryngitis $(p<0.01)$.

Conclusion: Etanercept and etanercept plus sulfasalazine are efficacious for the long-term management of patients with RA. The addition of etanercept or substitution with etanercept should be considered as treatment options for patients not adequately responding to sulfasalazine.

Several options including disease-modifying antirheumatic drugs (DMARD), such as methotrexate and sulfasalazine and anti-tumour necrosis factor (TNF) agents such as etanercept, infliximab and adalimumab, are available for the treatment of patients with rheumatoid arthritis (RA). For patients with an inadequate response to DMARD therapy, one recommended therapeutic option is anti-TNF therapy either added to or as a replacement for the existing regimen. ${ }^{1}$

In clinical studies, anti-TNF agents are highly effective and generally well tolerated when added to existing treatment regimens for patients with active RA who do not respond to a DMARD such as methotrexate ${ }^{3-8}$ or sulfasalazine, another frequently prescribed DMARD. However, there are very few studies assessing the benefits and risks of adding an anti-TNF agent to existing sulfasalazine therapy for patients with RA inadequately responding to sulfasalazine. ${ }^{9-12}$ Combe et al ${ }^{10}$ previously reported on the 6-month interim results from the current study; the 6-month results showed that etanercept, in combination with or in place of sulfasalazine, resulted in substantial improvements in RA. ${ }^{10}$ Both etanercept regimens were well tolerated. ${ }^{10}$

This 2-year report provides data on the longterm therapeutic response including patientreported outcomes (PRO) and safety of etanercept, added to or in place of sulfasalazine, versus sulfasalazine alone in patients with active RA, despite stable sulfasalazine therapy.

\section{PATIENTS AND METHODS}

\section{Study design and patients}

This was a 2-year randomised, double-blind, doubledummy, multicentre study in patients with active RA who had an inadequate response to sulfasalazine.

Eligible patients were 18 years of age or older with disease duration of 20 years or less with active adultonset RA (functional class I-III), defined as six or more swollen and 10 or more tender joints and one or more of the following: erythrocyte sedimentation rate (ESR; Westergren) $\geqslant 28 \mathrm{~mm}$ at the end of the first hour; serum C-reactive protein $\geqslant 20 \mathrm{mg} / \mathrm{l}$ and morning stiffness for 45 minutes or longer. Patients must have received stable doses of sulfasalazine (2$3 \mathrm{~g}$ daily) for 4 months or more before screening. Details of the exclusion/inclusion criteria have been published previously. ${ }^{10}$

This study was conducted in accordance with the International Conference on Harmonisation guidelines for good clinical practice in the European Community and the Declaration of Helsinki. Ethics committees of the participating centres approved the study protocol. Patients gave written informed consent before participating in the study.

\section{Treatment}

Patients were randomly assigned to one of three treatment groups (in a $2: 1: 2$ ratio): etanercept (etanercept $25 \mathrm{mg}$ by subcutaneous injection twice 


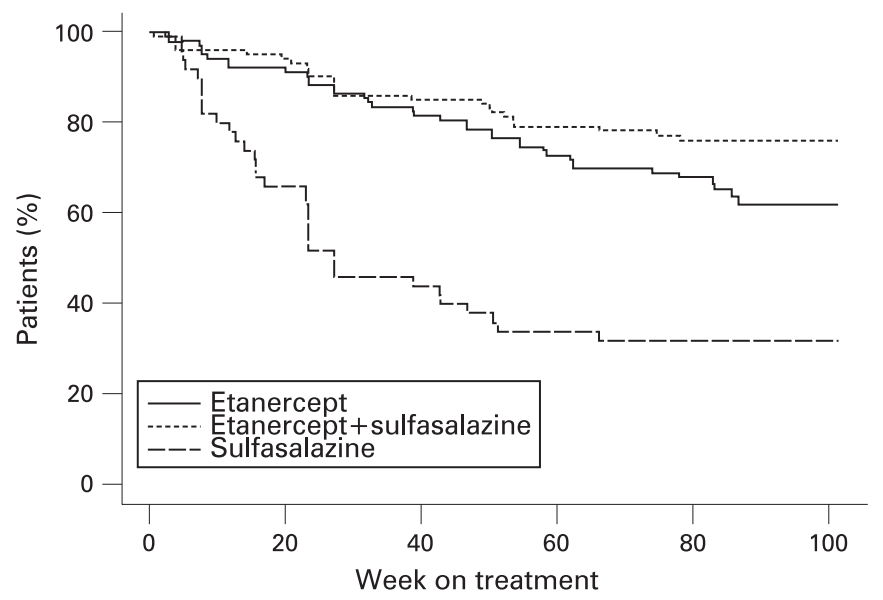

Figure 1 Percentage of patients remaining in the study versus time (in weeks). Based on the log-rank test, the $p$ values for the comparisons of the time to discontinuation are $<0.001$ (sulfasalazine versus etanercept), $<0.001$ (sulfasalazine versus combination), and 0.06 (etanercept versus combination).

weekly plus placebo); sulfasalazine (sulfasalazine $2,2.5$, or $3 \mathrm{~g}$ daily plus placebo) or combination (etanercept plus sulfasalazine) therapy. Patients in the etanercept group discontinued sulfasalazine at baseline.

\section{Clinical assessment}

Response to therapy was assessed at baseline and at weeks 2, 4, 8, $12,16,20,24,32,40,48,56,68,80,92$ and 104 . The primary efficacy endpoint, the percentage of patients achieving $20 \%$ or greater improvement, as defined by American College of Rheumatology (ACR) 20 criteria, ${ }^{13}$ at week 24 , was reported previously.

Key efficacy assessments included ACR response rates (ACR 20, ACR 50 and ACR 70), disease activity score (DAS) and morning stiffness in minutes. Assessments were based on ACR criteria and DAS as previously reported. ${ }^{10}$

PRO measures included the health assessment questionnaire (HAO) disability index, ${ }^{14}{ }^{15}$ EQ-5D visual analogue scale (VAS), ${ }^{16}$ patient global assessment of overall RA activity (PGAD) and patient general health assessment (GHVAS). The PGAD is recorded on an 11-point numeric rating scale in which a score of zero means no disease activity and a score of 10 means extreme disease activity. For the GHVAS, patients responded to the question "How do you feel concerning your arthritis?" by marking the appropriate position on a 100-mm VAS ranging from "very well" to "extremely bad." For both PGAD and GHVAS, lower scores imply better health.

To determine whether PRO closely reflected clinical improvements, the study examined the relationship between changes in health status measures or disability and changes in disease activity. Pearson correlation analysis was performed between the four PRO measures (HAO, EQ-5D VAS, PGAD and GHVAS) and a measure of disease activity, DAS.

\section{Safety assessments}

Safety assessments were based on reports of adverse events and results of routine physical examinations and laboratory determinations. An event was considered to be a treatment-emergent adverse event (TEAE) if it occurred during the study or if the severity or frequency of a preexisting event increased during the study. A serious adverse event (SAE) included any event resulting

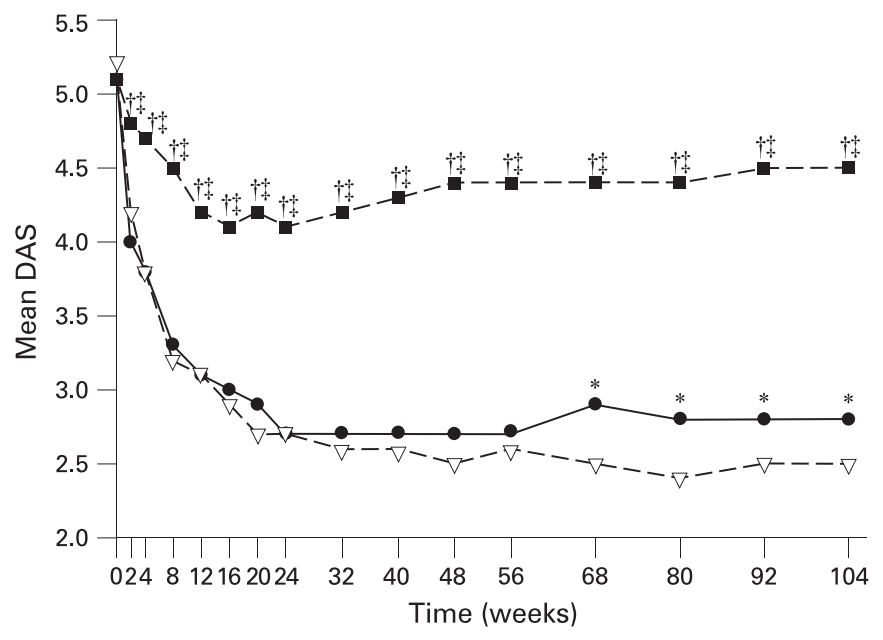

Figure 2 Mean disease activity score (DAS) over time (in weeks; last-observation-carried-forward, modified intent-to-treat analysis). $\nabla$ Etanercept plus sulfasalazine; - etanercept; $\square$ sulfasalazine. ${ }^{*} p<0.05$ etanercept versus combination; ${ }^{\dagger} p<0.05$ sulfasalazine versus combination; $\mathrm{p}<0.05$ sulfasalazine versus etanercept.

in death, hospitalisation or cancer. An infection was a serious infection if reported as an SAE.

Testing for antinuclear, anti-double-stranded DNA, antietanercept and anti-cardiolipin antibodies was performed at screening and at specified visits.

The incidence of malignancies in this study was compared with the incidence estimated from the US National Cancer Institute's (NCI) surveillance, epidemiology, and end results (SEER) database. ${ }^{17}$ The age and sex-specific incidence rates for cancer from the SEER database were applied to the exposure in this study.

\section{Statistical analysis}

The ACR 20, ACR 50 and ACR 70 response rates were analysed using the Mantel-Haenszel $\chi^{2}$ test, stratified by study centre. Changes from baseline in components of the ACR and PRO (HAO disability index, EQ-5D, GHVAS and PGAD) were analysed with a two-way analysis of covariance with treatments and centre as factors and the baseline as a covariate. Efficacy analyses were based on a modified intent-to-treat population, including patients who received any test article and provided efficacy data at baseline and at any subsequent visit. The proportions of patients with clinically meaningful changes in the HAO disability index at week 104 were compared between treatment groups using $\chi^{2}$ analyses. Pearson correlation analyses were used to assess correlations between DAS and PRO over 104 weeks. The last-observation-carried-forward (LOCF) approach was used to account for missing data points. LOCF imputation was also applied to patients who discontinued for unsatisfactory response. Adverse events were summarised and compared among treatment groups, using $\chi^{2}$ or Fisher's exact test. The sample sizes, 100 for the etanercept-alone group and sulfasalazine plus etanercept group versus 50 for the sulfasalazine-alone group, gave approximately $90 \%$ power to detect pairwise differences in the ACR 20 response of $33 \%$ versus $66 \%$ between the monotherapies.

\section{RESULTS}

\section{Efficacy}

Of the 260 patients who were randomly assigned in the study, 254 patients received one or more test articles (etanercept 103, 

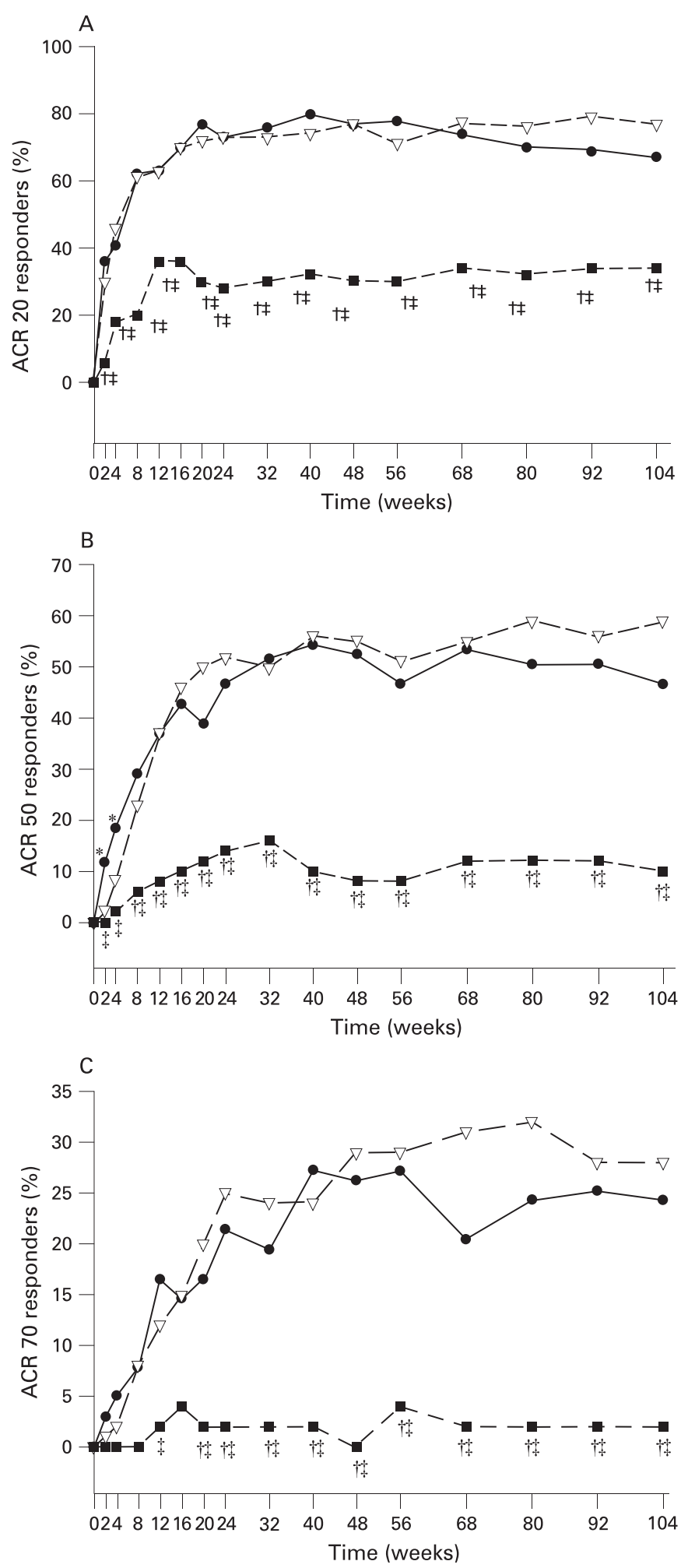

Figure 3 Percentage of patients in each treatment group achieving an American College of Rheumatology (ACR) response (last-observationcarried-forward). (A) ACR 20; (B) ACR 50 and (C) ACR 70. $\nabla$ Etanercept plus sulfasalazine; etanercept; $\square$ sulfasalazine. ${ }^{*} p<0.01$ etanercept versus combination; ${ }^{\dagger} \mathrm{p}<0.01$ sulfasalazine versus combination; ${ }^{*} \mathrm{p}<0.05$ sulfasalazine versus etanercept.

sulfasalazine 50 and combination 101). ${ }^{10}$ The population was predominantly white, female, and had a mean age of 51 years (consistent with the typical RA population). As previously reported, there were no significant differences among the groups

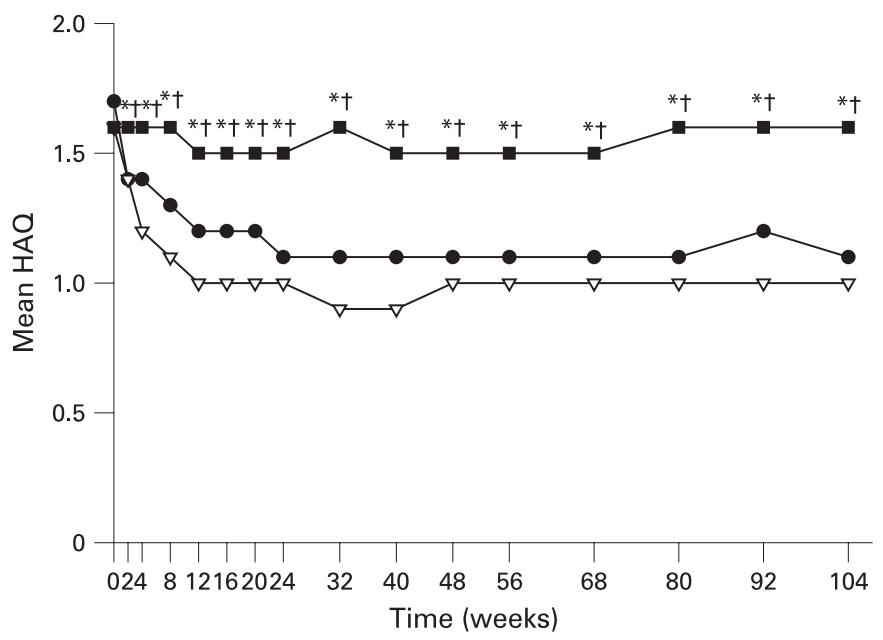

Figure 4 Mean health assessment questionnaire (HAO) scores from baseline to week 104 for patients with rheumatoid arthritis receiving sulfasalazine (SSZ), etanercept (ETN), or combination therapy with sulfasalazine and etanercept (last-observation-carried-forward analysis). $\nabla$ Etanercept plus sulfasalazine; 0 etanercept; $\square$ sulfasalazine. ${ }^{*} p<0.01$ sulfasalazine versus etanercept; ${ }^{\dagger} p<0.01$ sulfasalazine versus combination.

in the baseline characteristics with the exception of the percentage of patients receiving previous corticosteroids and the mean number of previous DMARD. ${ }^{10}$

A total of 96 patients discontinued the study: 38 receiving etanercept, 34 receiving sulfasalazine and 24 receiving combination therapy. Overall, a significantly greater number of the patients who continued on sulfasalazine monotherapy (68\%) withdrew from the study compared with those receiving etanercept, either as combination $(24 \%)$ or replacement $(37 \%)$ therapy $(p<0.001)$; the difference between etanercept and the combination was also significant $(p<0.05)$. The most common primary reason for discontinuation, lack of efficacy, led to a significantly higher number of withdrawals in patients receiving sulfasalazine $(52 \%)$ compared with those receiving either etanercept regimen ( $6 \%$ for each; $p<0.001)$. Discontinuations because of adverse events were not significantly different among the groups $(8 \%, 19 \%$ and 10\%, for the sulfasalazine, etanercept and combination groups, respectively) and there was no trend in the types of adverse events that led to discontinuation in any of the groups.

Times to discontinuation were estimated using the KaplanMeier method (fig 1); the combination group had the longest times to discontinuation. Based on the log-rank test, the differences among the three groups were statistically significant $(p<0.001$ for sulfasalazine vs etanercept or combination and $p=0.06$ for etanercept vs the combination).

Disease activity, as assessed by mean DAS, was significantly lower in the groups receiving etanercept than in the group receiving sulfasalazine from week 2 to week $104(p<0.01$, fig 2$)$. Significantly lower mean DAS values were observed during weeks 68-104 for the combination group compared with the etanerceptalone group $(p<0.05)$. A significantly higher proportion of patients receiving the combination or etanercept had a low level of disease activity (as assessed by DAS <2.4) compared with those receiving sulfasalazine after 6 months, which was maintained throughout the 2 years $(p<0.01)$; at 2 years, $57.0 \%$ of patients receiving combination, $45.6 \%$ receiving etanercept and $4.0 \%$ receiving sulfasalazine. Likewise, the proportion of patients achieving 

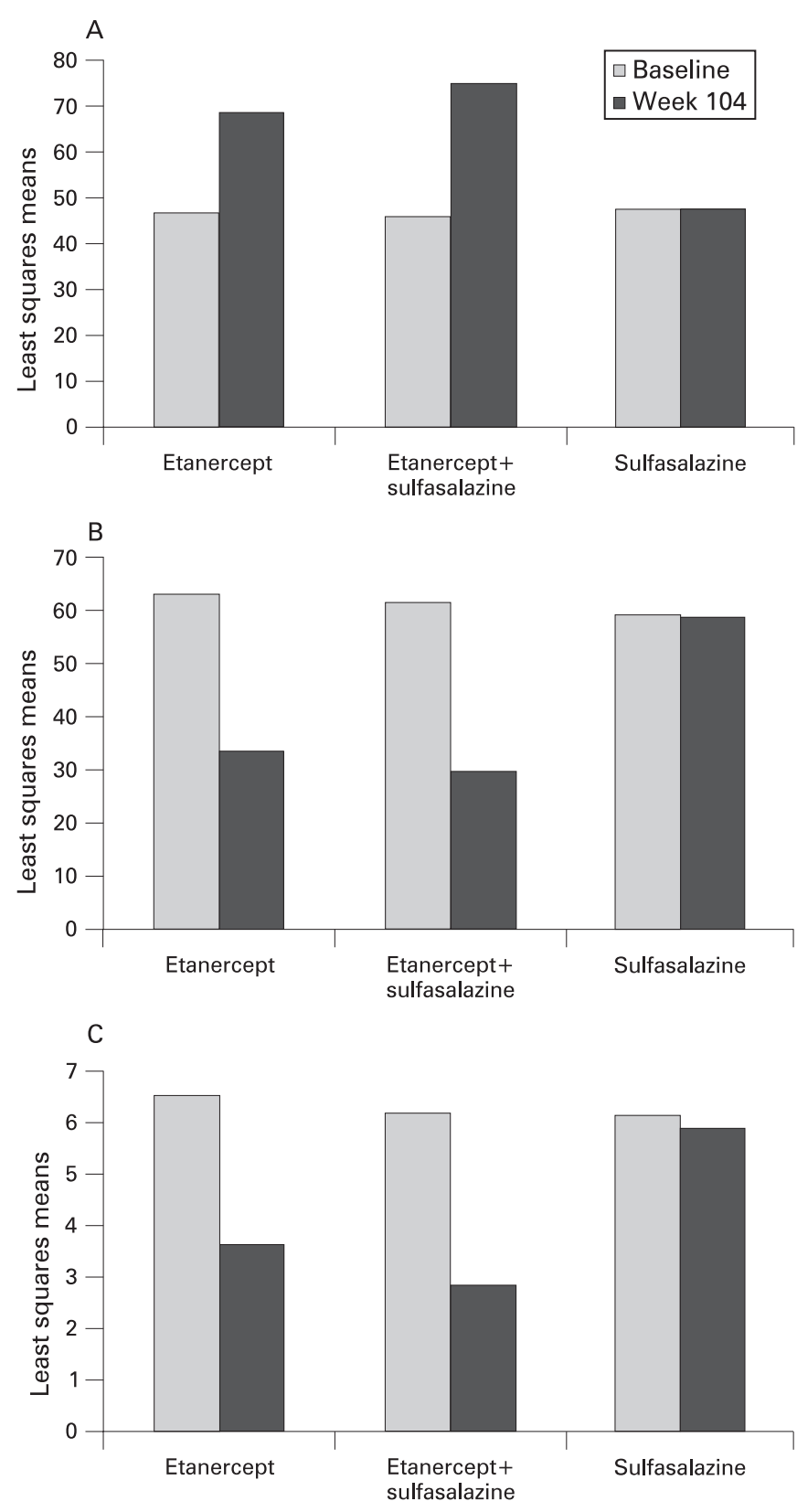

Figure 5 Health status at baseline and week 104 for patients with rheumatoid arthritis receiving etanercept, sulfasalazine, or combination therapy as measured by least squares means for EQ-5D, patient general health assessment (GHVAS) and global assessment of overall rheumatoid arthritis activity (PGAD) (last-observation-carried-forward analysis). (A) EQ-5D; (B) GHVAS; (C) PGAD.

disease remission was significantly higher with the etanercept groups compared with the sulfasalazine group $(p<0.01)$.

Treatment response as assessed by ACR 20 was achieved by a significantly higher percentage of patients receiving etanercept, added to or in place of sulfasalazine, compared with those receiving sulfasalazine $(p<0.01$; fig $3 A)$. Similarly, these significant differences in treatment response among the groups were also seen using ACR $50(p<0.01)$ and ACR $70(p<0.01$; fig $3 B, C$ ) criteria. For ACR 20 responses, the differences were significant beginning at week 2; for ACR 50 and ACR 70, the differences were significant beginning at weeks 8 and 12, respectively. Response rates were not significantly different between the two groups receiving etanercept.
The early and sustained response pattern was also seen with components of the ACR response criteria. Significant differences $(p<0.01)$ were seen for combination therapy or etanercept alone compared with sulfasalazine alone. Comparing the etanercept groups, the combination group separated from the etanercept monotherapy group for a short period (weeks 8 to 20) as demonstrated by the lower ESR levels $(p<0.05)$. No significant difference between these groups was seen for other ACR components before week 68 (total swollen joints) or week 92 (patient global assessment and physician global assessment), after which time the combination group showed better responses $(p<0.05)$.

\section{Patient-reported outcomes}

Mean $\mathrm{HAO}$ values for the etanercept and combination groups were significantly lower than those for the sulfasalazine group from week 2 ( $p<0.01$, sulfasalazine vs etanercept or combination) and these differences were sustained for the remainder of the 2-year study ${ }^{10}(p<0.01$; fig 4$)$.

Three health status measures using least squares means at week 104 are presented in fig 5. Patients who received etanercept or combination therapy showed significantly lower GHVAS scores, indicative of better general health, than patients who received sulfasalazine at week 104. Likewise, recipients of etanercept or combination therapy reported significantly better health states, as indicated by lower RA activity (PGAD) and higher EQ-5D scores than patients receiving sulfasalazine.

Our analyses also found that a significantly higher proportion of patients receiving etanercept or the combination attained the threshold of HAQ improvement $\geqslant 0.22$ by week 104 compared with those receiving sulfasalazine $(p<0.01$ compared with sulfasalazine alone). Similarly, a significantly higher proportion of patients receiving etanercept or the combination achieved EQ-5D VAS scores above population norms ${ }^{18}$ at week 104 compared with those receiving sulfasalazine $(\mathrm{p}<0.01$ compared with sulfasalazine alone).

Further analyses revealed that changes in disease activity from baseline to 104 weeks correlated with changes in disability as measured by the HAQ disability index and with changes in health status measures. Pearson correlations between the change from baseline in DAS and HAQ (0.57), PGAD (0.69), EQ-5D VAS (-0.61), or GHVAS (0.67) were all significant $(\mathrm{p}<0.001)$.

\section{Safety}

The pattern of adverse events reported during the 2 years of the study was not different from that reported during the first 6 months of the study. ${ }^{10}$ There were no significant differences between the combination and either monotherapy groups in the overall incidence of non-infectious adverse events (table 1). However, there were significantly more treatment-emergent infections in patients receiving etanercept than in those receiving sulfasalazine $(\mathrm{p}<0.001)$.

Treatment-emergent infections per patient-year were significantly higher with etanercept (1.72) than with etanercept and sulfasalazine (1.11) or with sulfasalazine $(0.86$; $p<0.001$ overall). There were significant differences between the etanercept group and both the sulfasalazine and combination groups $(p<0.001)$. Medically important infections occurred in more patients receiving etanercept $(11 ; 10.7 \%)$ than in those receiving combination (5; $5 \%, \mathrm{p}$ not significant) or sulfasalazine $(0$; $\mathrm{p}<0.05)$. After 352 days receiving combination therapy, one patient was hospitalised and withdrawn from the study and 
Table 1 Number (\%) of patients with the most common TEAE ( $\geqslant 10 \%$ in any treatment group)

\begin{tabular}{|c|c|c|c|}
\hline Body system TEAE & $\begin{array}{l}\text { Etanercept } \\
(\mathrm{n}=103)\end{array}$ & $\begin{array}{l}\text { Etanercept }+ \\
\text { sulfasalazine } \\
(\mathrm{n}=101)\end{array}$ & $\begin{array}{l}\text { Sulfasalazine } \\
(\mathrm{n}=50)\end{array}$ \\
\hline \multicolumn{4}{|l|}{ Non-infectious adverse events } \\
\hline Any TEAE (excluding infection) & $90(87.4)$ & $80(79.2)$ & $32(64.0)^{*}$ \\
\hline Injection site reaction & $34(33.0)$ & $21(20.8)$ & $2(4.0)^{*}+$ \\
\hline Headache & $11(10.7)$ & $25(24.8) \dagger$ & $4(8.0) \div$ \\
\hline Back pain & $9(8.7)$ & $20(19.8)$ & $5(10.0)$ \\
\hline Nausea & $7(6.8)$ & $19(18.8) \dagger$ & $5(10.0)$ \\
\hline Accidental injury & $16(15.5)$ & $17(16.8)$ & $1(2.0)^{*}+$ \\
\hline Asthenia & $5(4.9)$ & $16(15.8) \dagger$ & $1(2.0) \ddagger$ \\
\hline Rash & $15(14.6)$ & $8(7.9)$ & $3(6.0)$ \\
\hline Dyspepsia & $14(13.6)$ & $12(11.9)$ & $2(4.0)$ \\
\hline Abdominal pain & $14(13.6)$ & $12(11.9)$ & $1(2.0)$ \\
\hline Injection site haemorrhage & $9(8.7)$ & $14(13.9)$ & $3(6.0)$ \\
\hline Arthralgia & $8(7.8)$ & $14(13.9)$ & $7(14.0)$ \\
\hline Rheumatoid arthritis & $10(9.7)$ & $12(11.9)$ & $5(10.0)$ \\
\hline Diarrhoea & $11(10.7)$ & $6(5.9)$ & $0.0^{*}$ \\
\hline Cough increased & $7(6.8)$ & $12(11.9)$ & $4(8.0)$ \\
\hline Parasthesia & $4(3.9)$ & $11(10.9)$ & $1(2.0)$ \\
\hline \multicolumn{4}{|l|}{ Infectious adverse events } \\
\hline Any TEAE infection & $76(73.8)$ & $60(59.4) \dagger$ & $21(42.0) *$ \\
\hline Upper respiratory tract infection & $29(28.2)$ & $29(28.7)$ & $10(20.0)$ \\
\hline Pharyngitis/laryngitis & $24(23.3)$ & $10(9.9)$ & $3(6.0)^{*}+$ \\
\hline Bronchitis & $21(20.4)$ & $12(11.9)$ & $4(8.0)$ \\
\hline Flu syndrome & $18(17.5)$ & $12(11.9)$ & $2(4.0)$ \\
\hline Gingival/dental infection & $7(6.8)$ & $12(11.9)$ & $2(4.0)$ \\
\hline Sinusitis & $12(11.7)$ & $3(3.0) \dagger$ & $0.0^{*}$ \\
\hline Miscellaneous skin infections§ & $19(18.4)$ & $12(11.9)$ & $0.0 *$ \\
\hline
\end{tabular}

treated with antituberculous drugs following a histological determination of tuberculous lymphadenitis.

There was no clustering of SAE. In the 2 years of the study, 23 patients receiving the combination, 27 receiving etanercept and two receiving sulfasalazine had one or more SAE. Noninfectious SAE were significantly greater in patients receiving etanercept $(20.8 \%$ for the combination and $20.4 \%$ for etanercept alone) compared with $4 \%$ for patients receiving sulfasalazine $(\mathrm{p}<0.01)$.

At all scheduled visits, patients receiving the combination had a significantly greater reduction in white blood cell (WBC) counts (mean change from baseline) than those receiving sulfasalazine alone $(p<0.001)$. In contrast, there was no significant difference in this laboratory parameter between the etanercept and sulfasalazine groups. Across all treatment groups, no subjects developed any NCI grade 3 or 4 WBC abnormalities, except for one subject who had a transient grade 4 WBC abnormality that normalised. All mean WBC counts were within normal limits at all visits.

Leucopenia was reported as a TEAE in eight (7.9\%) patients in the combination group, two $(1.9 \%)$ in the etanercept group and none in the sulfasalazine group ( $p$ not significant). None of the events of leucopenia were associated with NCI grade 3 or 4 levels of neutrophils or other NCI grade 3 or 4 laboratory abnormalities. No patients withdrew from the study because of TEAE of leucopenia; all events were resolved during the study.

Malignancy was diagnosed in two patients receiving etanercept. One patient with a history of skin cancer developed actinic squamous cell carcinoma of the lower lip. The second patient, whose diagnosis was myelodysplastic syndrome after 12 weeks of etanercept, then developed myelocytic leukaemia and subsequently died.

The incidence of malignancy observed in this study was compared with the expected incidence based on the US SEER programme. The observed number (1.0) of malignancies in etanercept or combination-treated patients during the entire study was lower than the expected numbers (2.7) based on SEER, which excludes non-melanoma skin cancers. ${ }^{17}$

The second reported death was a patient with interstitial pneumonitis, who had a history of smoking, coronary obstructive pulmonary disease and childhood pneumonia.

No patients developed systemic lupus erythematosus or a lupus-like syndrome, glomerulonephritis, pleuritis, peritonitis, or seizure. There were no reports of central demyelinating diseases in this study. Occurrences of auto-antibodies were not significantly different among groups at baseline and at the last visit.

\section{DISCUSSION}

The results from this 2-year double-blind, randomised study in patients with active RA despite sulfasalazine therapy provide further evidence that etanercept, either alone or when added to existing DMARD therapy, has a favourable long-term safety and efficacy profile. The addition of or switching to etanercept resulted in a rapid and sustained improvement of all clinical efficacy endpoints compared with sulfasalazine alone. The efficacy results were similar in both etanercept groups, indicating that patients inadequately responding to sulfasalazine would benefit by either the addition of etanercept to their existing (sulfasalazine) treatment or by switching from sulfasalazine to etanercept. Treatment with etanercept, sulfasalazine, or 
the combination did not result in any unexpected safety findings. The combination of etanercept and sulfasalazine was not associated with greater toxicity than either therapy alone.

Modifications to a less than adequate treatment regimen, such as the addition of another agent or substitution of a "failing" drug with another agent, are a standard approach. Clinical studies in patients with RA have shown that adding or substituting an anti-TNF agent to the existing methotrexate therapy ${ }^{371920}$ results in a significant improvement in the therapeutic response. The addition of an anti-TNF agent to (or in place of) inadequate sulfasalazine has not been extensively evaluated in clinical trials. In the only other placebo-controlled trial in RA patients not adequately treated with sulfasalazine, adalimumab was added to the existing treatment. ${ }^{9}{ }^{12}$. In that study, patients receiving the combination showed a greater degree of improvement than those receiving sulfasalazine alone. However, modest sample sizes (sulfasalazine alone, $\mathrm{n}=33$; sulfasalazine plus adalimumab, $\mathrm{n}=29$ ) make it difficult to compare the efficacy results among the treatment arms. In a larger open-label study evaluating adalimumab alone and in patients inadequately responding to at least one traditional DMARD, the number of ACR 20 responders was similar for adalimumab plus sulfasalazine and adalimumab alone. ${ }^{11}$

Patients who received etanercept monotherapy or etanercept plus sulfasalazine combination achieved significantly higher ACR response rates than patients in the sulfasalazine monotherapy group $(p<0.01)$. For all three treatment groups, the core components generally followed the pattern of the ACR composite response. Mean ESR values in both the etanercept plus sulfasalazine combination and etanercept monotherapy groups were significantly lower than in the sulfasalazine monotherapy group from week $2(p<0.01)$ to week 104 $(p<0.01)$. For a short period (week 8 to week 20) the combination group had significantly lower ESR levels than the etanercept-alone group $(p<0.05)$, but the difference was not significant from week 24 to week 104.

Physical function scores were significantly improved in the groups receiving etanercept, either alone or in combination with sulfasalazine, compared with sulfasalazine alone, using several previously defined thresholds. ${ }^{21}{ }^{22}$ In patients with RA, a change of 0.22 or more units in the HAQ score has been used to estimate the number of patients with a clinically significant improvement in physical function. ${ }^{22}$ In this study, by week 104, three in every four patients receiving etanercept, either alone or as add-on therapy, achieved a HAQ improvement of 0.22 or more, compared with less than 1 in two patients continuing on sulfasalazine therapy.

Etanercept and combination therapy resulted in significantly greater improvements in all measured PRO than the modest improvements seen with sulfasalazine therapy. Furthermore, there was a significant correlation between disease activity, as assessed by the DAS, and physical function, as assessed by the HAO. Significant correlation was also observed between disease activity and other PRO, including the EQ-5D VAS and PGAD.

The study was originally designed as a 6-month trial, which is not long enough to detect radiographic changes, but was subsequently extended to 2 years. However, because both disease activity and radiographically assessed joint damage have been shown to be major contributors to the physical functioning of the patient with RA, ${ }^{23-26}$ the significant improvements in disease activity and physical functioning would suggest that radiographic progression was inhibited in these patients.

Treatment with etanercept, sulfasalazine, or the combination did not result in any unexpected safety findings; however, the comparison of TEAE among treatment groups in this study has bias. Generally, with any drug patients report adverse events at a higher frequency during the first few weeks or months after the start of treatment and patients who cannot tolerate the drug are withdrawn. As a protocol-inclusion criterion, patients in the sulfasalazine group were required to have tolerated sulfasalazine well; hence, these patients were expected to report adverse events at a lower rate than patients receiving a "new" treatment.

The use of anti-TNF agents has also been associated with the increased frequency of autoantibodies, which could result in autoimmune diseases such as systemic lupus erythematosus. ${ }^{27-29}$ Although the number of patients who tested positive for antidsDNA increased by the end of our study, there were no relevant clinical symptoms associated with the positive antidsDNA test results in any of the treatment groups.

Leucopenia, neutropenia, thrombocytopenia and pancytopenia have also been reported in patients receiving anti-TNF therapies. In this study, the incidence of TEAE of leucopenia was greater in the combination group $(7.9 \%)$ than in the etanercept group $(1.9 \%)$ or the sulfasalazine group $(0 \%)$, but between-group differences were not statistically significant. Investigators categorised the severity of all eight events in the combination group as mild; none of the TEAE of leucopenia were associated with NCI grade 3 or 4 values of neutrophils or with other NCI grade 3 or 4 laboratory abnormalities. No patients withdrew from the study because of leucopenia. The lower WBC counts thus did not seem to be clinically relevant. Furthermore, there were no reports of aplastic anaemia or pancytopenia.

This study shows that the addition of etanercept to sulfasalazine, a DMARD other than methotrexate, can provide significant long-term improvement in efficacy. In particular, it shows that etanercept provides benefit when added to or switched with sulfasalazine in patients who show an inadequate response to sulfasalazine. The reported findings are especially relevant in view of the recent reports regarding monotherapy failures, which discourage the choice of both methotrexate plus sulfasalazine and leflunomide plus sulfasalazine combination therapy. ${ }^{30}$ Generally, combination treatment was not associated with an increased incidence of adverse events. Etanercept either alone or added to existing sulfasalazine therapy is associated with a favourable risk benefit profile, thus broadening the range of options for the treatment of patients with active RA.

Acknowledgements: The authors would like to acknowledge Ruth Pereira and $J$ Maitland Young in the Publications and External Communications Group at Wyeth for their writing support and S Sun of Wyeth Research for contributions to statistical analyses of the study.

Funding: Supported by Wyeth Research, Collegeville, PA, USA (study drug and grants to investigational sites).

Competing interests: Declared. BC was a consultant and a speaker for Wyeth. CC received investigator fees for carrying out Wyeth trials. UF received reimbursement from Wyeth Italia for running educational programmes. MG was reimbursed by Wyeth for attending several conferences and was paid for giving educational talks. PPG received support for clinical studies from Wyeth Research. TKK was a consultant and a speaker for Wyeth and received funds for research. JW, RK, AS and SF are employees of Wyeth Research.

Ethics approval: Ethics committees of the participating centres approved the study protocol.

Patient consent: Obtained.

List of the Etanercept Study 309 Investigators in Europe and Australia (other than the authors): Professor Maxime Dougados, Hopital Cochin, Paris, France;

Professor Joël Dehais, Hopital Pellegrin, Bordeaux, France; Professor Philippe Goupille, Hopital Trousseau, Tours, France; Professor Pierre Miossec, Hopital E Herriot, Lyon, 
France; Dr Anett Grässler, University Hospital, Dresden, Germany; Professor Umberto Ambanelli, Universita di Parma, Parma, Italy; Professor Silvano Todesco, Policlinico Universitario, Padova, Italy; Dr Hillary Capell, Glasgow Royal Infirmary, Glasgow, UK; Dr lan Griffiths, Freeman Hospital, Newcastle upon Tyne, UK; Dr Richard Hull, Queen Alexandra Hospital, Portsmouth, Hants, UK; Dr George Kitas, Corbett Hospital, Stourbridge, West Midlands, UK; Dr Robert Moots, Fazakerley Hospital, Liverpool, UK; Professor David GI Scott, Norfolk and Norwich Hospital, Norwich, UK; Professor David L Scott, Dulwich Hospital, London, UK; Dr Peter Sheldon, Leicester Royal Infirmary, Leicester, UK; Dr Bryan Williams, University Hospital of Wales, Cardiff, South Wales, UK; Dr Gary Wright, Musgrave Park Hospital, Belfast, UK; Dr Paresh Jobanputra, University of Birmingham, Birmingham, UK; Dr Knut Mikkelsen, Lillehammers Sanitetsforening, Lillehammer, Norway; Dr Olav Bjorneboe, Martina Hansens Hospital, Gjetterum, Norway; Dr Petr Vitek, Centrum Rehabilitace, Zlin, Czech Republic; Dr Ladislav Bortlik, NZZ Bormed, Ostrava, Czech Republic; Dr Marie Sedlackova, Thomayer University Hospital, Praha, Czech Republic; Dr Sevda Augustinova, Medipont, (Jerzy Lech) Ceske Budejovice, Czech Republic; Dr Peter Nash, Sixth Avenue Specialist Centre, Maroochydore, Australia; Dr Stephen Hall, Cabrini Medical Centre, Malvern, Australia; Dr Florin Radulescu, Ambulatoriul Centrului Metodologic de Reumatologie, Bucuresti, Romania; Dr Coman Tanasescu, Institutul de Medicina Interna, Bucuresti, Romania; Dr Horatiu Bolosiu, Spitalul Clinic Judetean Cluj, ClujNapoca, Romania.

\section{REFERENCES}

1. O'Dell JR. Therapeutic strategies for rheumatoid arthritis. N Engl J Med 2004;350:2591-602.

2. Furst DE, Breedveld FC, Kalden JR, Smolen JS, Burmester GR, Emery P, et al. Updated consensus statement on biological agents for the treatment of rheumatic diseases, 2006. Ann Rheum Dis 2006;657:iii2-iii15

3. Weinblatt ME, Kremer JM, Bankhurst AD, Bulpitt KJ, Fleischmann RM, Fox RI, et al. A trial of etanercept, a recombinant tumor necrosis factor receptor:Fc fusion protein, in patients with rheumatoid arthritis receiving methotrexate. $N$ Engl J Med 1999:340:253-9

4. Maini R, St Clair EW, Breedveld F, Furst D, Kalden J, Weisman M, et al. Infliximab (chimeric anti-tumour necrosis factor alpha monoclonal antibody) versus placebo in rheumatoid arthritis patients receiving concomitant methotrexate: a randomised phase III trial. ATTRACT Study Group. Lancet 1999;354:1932-9.

5. Matsumoto I, Lee DM, Goldbach-Mansky R, Sumida T, Hitchon CA, Schur PH, et al. Low prevalence of antibodies to glucose-6-phosphate isomerase in patients with rheumatoid arthritis and a spectrum of other chronic autoimmune disorders. Arthritis Rheum 2003:48:944-54.

6. Maini RN, Breedveld FC, Kalden JR, Smolen JS, Davis D, Macfarlane JD, et al. Therapeutic efficacy of multiple intravenous infusions of anti-tumor necrosis factor alpha monoclonal antibody combined with low-dose weekly methotrexate in rheumatoid arthritis. Arthritis Rheum 1998;41:1552-63.

7. Weisman MH, Moreland LW, Furst DE, Weinblatt ME, Keystone EC, Paulus HE, et al. Efficacy, pharmacokinetic, and safety assessment of adalimumab, a fully human antitumor necrosis factor-alpha monoclonal antibody, in adults with rheumatoid arthritis receiving concomitant methotrexate: a pilot study. Clin Ther 2003;25:1700-21.

8. Bankhurst AD. Etanercept and methotrexate combination therapy. Clin Exp Rheumatol 1999;17(6 Suppl 18):S69-72.

9. Furst DE, Schiff MH, Fleischmann RM, Strand V, Birbara CA, Compagnone D, et al. Adalimumab, a fully human anti tumor necrosis factor-alpha monoclonal antibody, and concomitant standard antirheumatic therapy for the treatment of rheumatoid arthritis: results of STAR (Safety Trial of Adalimumab in Rheumatoid Arthritis). J Rheumatol 2003;30:2563-71.

10. Combe B, Codreanu C, Fiocco U, Gaubitz M, Geusens PP, Kvien TK, et al. Etanercept and sulfasalazine, alone and combined, in patients with active rheumatoid arthritis despite receiving sulfasalazine: a double-blind comparison. Ann Rheum Dis 2006:65:1357-62.

11. Burmester GR, Mariette X, Montecucco C, Monteagudo-Saez I, Malaise M, Tzioufas $A G$, et al. Adalimumab alone and in combination with disease-modifying antirheumatic drugs for the treatment of rheumatoid arthritis in clinical practice: the Research in Active Rheumatoid Arthritis (ReAct) trial. Ann Rheum Dis 2007;66:732-9.

12. US Food and Drug Administration. Adalimumab product approval informationlicensing action. Updated 2005. http://www.fda.gov/cder/biologics/products/ adalabb123102.htm (accessed September 2008).
13. Felson DT, Anderson JJ, Boers M, Bombardier C, Furst D, Goldsmith C, et al. American College of Rheumatology. Preliminary definition of improvement in rheumatoid arthritis [comment]. Arthritis Rheum 1995;38:727-35.

14. Bruce B, Fries JF. The Stanford Health Assessment Questionnaire: a review of its history, issues, progress, and documentation. J Rheumatol 2003;30:167-78.

15. Gardiner PV, Sykes HR, Hassey GA, Walker DJ. An evaluation of the Health Assessment Questionnaire in long-term longitudinal follow-up of disability in rheumatoid arthritis. Br J Rheumatol 1993;32:724-8.

16. Hurst NP, Kind P, Ruta D, Hunter M, Stubbings A. Measuring health-related quality of life in rheumatoid arthritis: validity, responsiveness and reliability of EuroOol (EQ5D). Br J Rheumatol 1997;36:551-9.

17. Surveillance, Epidemiology, and End Results (SEER) Program. Public-use data (1973-1999) [11 Registries, 1992-1999]. National Cancer Institute, DCCPS, Surveillance Research Program, Cancer Statistics Branch, released April 2002, based on the November 2001 submission. http://www.SEER.cancer.gov (accessed Dec 2008).

18. Kind P, Hardman G, Macran S. UK Population Norms for EQ-5D. Discussion paper 172. Center for Health Economics, University of York, York, UK. November 1999. http://www.york.ac.uk/inst/che/publications/publicationsbyyear.htm (accessed 17 Dec 2008)

19. Keystone EC, Kavanaugh AF, Sharp JT, Tannenbaum H, Hua Y, Teoh LS, et al. Radiographic, clinical, and functional outcomes of treatment with adalimumab (a human anti-tumor necrosis factor monoclonal antibody) in patients with active rheumatoid arthritis receiving concomitant methotrexate therapy: a randomized, placebo-controlled, 52-week trial. Arthritis Rheum 2004;50:1400-11.

20. van Riel PL, Taggart AJ, Sany J, Gaubitz M, Nab HW, Pedersen R, et al. Efficacy and safety of combination etanercept and methotrexate versus etanercept alone in patients with rheumatoid arthritis with an inadequate response to methotrexate: the ADORE study. Ann Rheum Dis 2006;65:1478-83.

21. Krishnan E, Sokka T, Hakkinen A, Hubert H, Hannonen P. Normative values for the Health Assessment Questionnaire disability index: benchmarking disability in the general population. Arthritis Rheum 2004;50:953-60.

22. Wells GA, Tugwell P, Kraag GR, Baker PR, Groh J, Redelmeier DA. Minimum important difference between patients with rheumatoid arthritis: the patient's perspective. J Rheumatol 1993;20:557-60.

23. Drossaers-Bakker KW, de Buck M, van Zeben D, Zwinderman AH, Breedveld FC, Hazes JM. Long-term course and outcome of functional capacity in rheumatoid arthritis: the effect of disease activity and radiologic damage over time. Arthritis Rheum 1999;42:1854-60.

24. Jansen LM, van Schaardenburg D, van Der Horst-Bruinsma IE, Bezemer PD, Dijkmans BA. Predictors of functional status in patients with early rheumatoid arthritis. Ann Rheum Dis 2000;59:223-6.

25. van der Heijde D, Landewe R, van Vollenhoven R, Fatenejad S, Klareskog L. Radiographic damage and 2-year radiographic progression determine physical function in patients with rheumatoid arthritis: a longitudinal analysis of the TEMPO trial. Abstract SAT0193. Amsterdam. The Netherlands: European League Against Rheumatism (EULAR), 2006

26. Odegard S, Landewe R, van der Heijde D, Kvien TK, Mowinckel P, Uhlig T. Association of early radiographic damage with impaired physical function in rheumatoid arthritis: a ten-year, longitudinal observational study in 238 patients. Arthritis Rheum 2006;54:68-75.

27. Eriksson C, Engstrand S, Sundqvist KG, Rantapaa-Dahlqvist S. Autoantibody formation in patients with rheumatoid arthritis treated with anti-TNF alpha. Ann Rheum Dis 2005;64:403-7.

28. De Rycke L, Kruithof E, Van Damme N, Hoffman IE, Van den Bossche N, Van den Bosch F, et al. Antinuclear antibodies following infliximab treatment in patients with rheumatoid arthritis or spondylarthropathy. Arthritis Rheum 2003;48:1015-23.

29. Palucka AK, Blanck JP, Bennett L, Pascual V, Banchereau J. Cross-regulation of TNF and IFN-alpha in autoimmune diseases. Proc Natl Acad Sci U S A 2005;102:3372-7.

30. Schoels M, Kapral T, Stamm T, Smolen JS, Aletaha D. Step-up combination versus switching of non-biological disease-modifying antirheumatic drugs in rheumatoid arthritis: results from a retrospective observational study. Ann Rheum Dis 2007:66:1059-65.

31. van der Kooij SM, de Vries-Bouwstra JK, Goekoop-Ruiterman YP, van Zeben D, Kerstens PJ, Gerards AH, et al. Limited efficacy of conventional DMARDs after initial methotrexate failure in patients with recent onset rheumatoid arthritis treated according to the disease activity score. Ann Rheum Dis 2007;66:1356-62. 